\title{
Maximum Likelihood Estimation
}

National Cancer Institute

\section{Source}

National Cancer Institute. Maximum Likelihood Estimation. NCI Thesaurus. Code C53331.

A method for estimating population characteristics based on limited data by choosing the sample values that make the data most likely to represent the general population. 